\title{
New approach to phylogenetic analysis of the genus Bifidobacterium based on partial HSP60 gene sequences
}

China General Microorganism Culture Collection Center, Institute of Microciology, Chinese Academy of Sciences, Beijing 100080, PR China

\author{
Wenying Jian, Lin Zhu and Xiuzhu Dong
}

Author for correspondence: Xiuzhu Dong. Tel: +8610625 58320. Fax: +861062558320. e-mail: dongxz@sun.im.ac.cn

\begin{abstract}
The partial 60 kDa heat-shock protein (HSP60) genes of 36 Bifidobacterium strains representing $\mathbf{3 0}$ different Bifidobacterium species and subspecies and of the type strain of Gardnerella vaginalis were cloned and sequenced using a pair of universal degenerate HSP60 PCR primers. The HSP60 DNA sequence similarities were determined for the taxa at various ranks as follows: 99.4-100\% within the same species, $96 \%$ at the subspecies level, and 73-96\% (mean $85 \%$ ) at the interspecies level (and $98 \%$ in the case of two groups of closely related species, Bifidobacterium animalis and Bifidobacterium lactis, Bifidobacterium infantis, Bifidobacterium longum and Bifidobacterium suis, whose 165 rRNA sequence similarities are all above 99\%). The HSP60 DNA sequence similarities between different Bifidobacterium species and $G$. vaginalis, a closely related bacterium according to $16 \mathrm{~S}$ rRNA analysis, ranged from 71 to $79 \%$ (mean $75 \%$ ). Although the topology of the phylogenetic tree constructed using the HSP60 sequences determined was basically similar to that for 165 rRNA, it seemed to be more clear-cut for species delineation, and the clustering was better correlated with the DNA base composition ( $\mathrm{mol} \%$ $G+C)$ than that of the 165 rRNA tree. In the HSP60 phylogenetic tree, all of the high-G+C (55-67 mol\%) bifidobacteria were grouped into one cluster, whereas the low-G+C species Bifidobacterium inopinatum (45 mol\%) formed a separate cluster with G. vaginalis (42 $\mathrm{mol} \%$ ) and Bifidobacterium denticolens ( $55 \mathrm{~mol} \%$ ); a Bifidobacterium species of intermediate $\mathrm{G}+\mathrm{C}$ content formed another cluster between the two. This study demonstrates that the highly conserved and ubiquitous HSP60 gene is an accurate and convenient tool for phylogenetic analysis of the genus Bifidobacterium.
\end{abstract}

Keywords: Bifidobacterium, HSP60 gene sequences, 16S rRNA sequences, phylogenetic analysis

\section{INTRODUCTION}

Members of the genus Bifidobacterium are Grampositive, anaerobic, non-motile bacteria. The cells are pleomorphic, irregular, non-spore-forming rods. Uniquely, they possess fructose-6-phosphate phosphoketolase and produce acetic and lactic acid as the endproducts of glucose metabolism. The habitats of bifidobacteria range from sewage to the intestines of humans, animals and honey bees (Scardovi, 1986), so they are believed to be predominantly a constituent of the intestinal flora. In recent years, new species of bifidobacteria have also been isolated from human dental caries (Crociani et al., 1996), fermented milk
(Meile et al., 1997) and an anaerobic digester (Dong et al., 2000). So far, 32 species, including two subspecies, have been assigned to this genus.

Miyake et al. (1998) analysed the 16S rRNA sequences of type strains of 31 Bifidobacterium species and of Gardnerella vaginalis. The result indicated that all of the species formed a compact cluster involving similarity levels ranging from 93 to $99 \%$, except for the two species Bifidobacterium inopinatum and Bifidobacterium denticolens, which have relatively low $\mathrm{G}+\mathrm{C}$ contents (45 and $55 \mathrm{~mol} \%$, respectively). For some species, the 16S rRNA sequence similarities were even above $99 \%$. So, although 16 S rRNA sequence-hom- 
ology analysis is demonstrated to be a useful tool for bacterial phylogenetic study, it cannot provide sufficient resolution to discriminate between closely related bacterial strains (Stackebrandt \& Goebel, 1994).

Lauer \& Kandler (1983) determined that DNA-DNA hybridization was an effective standard for species designation within the genus Bifidobacterium, and that DNA-DNA homology would be $70 \%$ or higher for the members within the same species. However, DNADNA hybridization is a laborious and inconvenient process. To determine the taxonomic position of a bacterial stain, one has to perform hybridizations between this strain and all the existing members of the genus. To study the relationship between different strains, even more pairwise hybridizations must be performed. In addition, the results of DNA-DNA hybridizations might not be as stable and comparable as those involving nucleotide sequences, because of the different experimental methods and conditions involved.

Thus, both approaches have limitations with respect to the phylogenetic analysis of bifidobacteria. In recent years, some housekeeping proteins such as the $60 \mathrm{kDa}$ heat-shock protein (HSP60) (Viale et al., 1994) and elongation factor (Kamla et al., 1996), because of their highly conserved primary structures and ubiquity, have been considered as useful phylogenetic markers. HSP60 belongs to the chaperonin family, members of which are present in all bacteria and eukaryotic cell organelles such as mitochondria and chloroplasts. These chaperonins facilitate the intracellular folding and assembly (into oligomeric complexes) of various polypeptide chains. Viale et al. (1994) and Gupta (1995) have previously observed that evolutionary trees constructed from the protein sequences of these molecules in eubacteria demonstrated remarkable similarity to those derived from $16 \mathrm{~S}$ rRNA genes. It is also known that almost all bacterial species have a single copy of the HSP60 gene in their genomes (Segal \& Ron, 1996). Thus, it is hypothesized that the HSP60 gene is not easily transferred from one bacterium to another, and is a suitable macromolecule for the phylogenetic study of closely related bacterial species and strains. Kwok et al. (1999) cloned and sequenced the partial HSP60 protein genes of 36 validly described strains representing 28 different staphylococcal species or subspecies within this genus. The results showed that the partial HSP 60 gene provided a convenient and accurate tool for species-specific identification and phylogenetic analysis of staphylococci.

In this study, a fragment (of approximately $0.6 \mathrm{~kb}$ ) of the HSP60 gene of 36 Bifidobacterium strains, including 31 type strains of different Bifidobacterium species or subspecies, five bifidobacterial isolates, and a type strain of the closely related micro-organism $G$. vaginalis were amplified, cloned and sequenced. The phylogenetic analysis based on these sequences showed that the partial HSP60 DNA sequence was a powerful tool for phylogenetic analysis of the genus Bifidobacterium.

\section{METHODS}

Bacterial strains. Thirty-seven bacterial strains, including 31 type strains of Bifidobacterium species, five bifidobacterial strains isolated from commercial dairy products, and one type strain of G. vaginalis were used in this study (Table 1). The strains were kindly provided by the Japan Culture Collection of Microorganism (JCM), the Deutsche Sammlung von Mikroorganismen und Zellkulturen (DSMZ), the American Type Culture Collection (ATCC), the China General Micro-organism Culture Collection Center, or were previously isolated and identified in our laboratory.

The strains were routinely grown in tryptone peptone yeastextract glucose medium (Scardovi, 1986). The media were prepared in glass tubes sealed with butyl rubber stoppers and screw caps. The gas phase was $\mathrm{N}_{2}$. After inoculation, tubes were incubated anaerobically at $37^{\circ} \mathrm{C}$.

DNA extraction. The chromosomal DNA was extracted using a modified version of the method of Marmur (1961). As the cell walls of bifidobacteria were hard to lyse with lysozyme and SDS, $0.5 \mathrm{mg}$ penicillin $\mathrm{G} \mathrm{ml}^{-1}$ culture was added to the cultures at the late exponential phase to assist cell breakage. The cultures were incubated for another 16-20 h. Proteinase $\mathrm{K}\left(100 \mu \mathrm{g} \mathrm{ml}{ }^{-1}, 37^{\circ} \mathrm{C}\right.$ for $1 \cdot 5-2 \mathrm{~h}$.) was used instead of phenol to remove the protein contamination.

PCR amplification. The HSP60 degenerate PCR primers, designed on the basis of the conserved protein sequences GDGTTATV and AVKAPGFGD in HSP60 (Rusanganwa et al., 1992), were H60F and H60R. The nucleotide sequences of $\mathrm{H} 60 \mathrm{~F}$ and $\mathrm{H} 60 \mathrm{R}$ were $5^{\prime}-\mathrm{GG}$ (ATGC) GA (CT) GG (ATGC) AC (ATGC) AC (ATGC) AC (ATGC) GC (ATGC) AC (ATGC) GT-3' and 5'-TC (ATGC) CC (AG) AA (ATGC) CC (ATGC) GG (ATGC) GC (CT) TT (ATGC) AC (ATGC) GC-3', respectively. The $25 \mu 1$ PCR mixture consisted of approximately $300 \mathrm{ng}$ DNA template, $2.5 \mathrm{nmol}$ each dNTP, $2.5 \mu \mathrm{l} \mathrm{Taq}$ DNA polymerase, $10 \times$ buffer, 50 pmol each of H60F and H60R (Sangon) and 2.5 U recombinant Taq DNA polymerase (TaKaRa). The PCR thermal cycling conditions were as follows: $94{ }^{\circ} \mathrm{C}$ for $5 \mathrm{~min}$, followed by 30 cycles of $94^{\circ} \mathrm{C}$ for $30 \mathrm{~s}, 55^{\circ} \mathrm{C}$ for $30 \mathrm{~s}$ and $72{ }^{\circ} \mathrm{C}$ for $1 \mathrm{~min}$. Finally, the reaction was maintained at $72^{\circ} \mathrm{C}$ for $10 \mathrm{~min}$. The PCR amplification was performed with a Thermolyne Amplitron I (Barnstead Thermolyne). After amplification, the reaction mixtures were electrophoresised through a $1 \%(\mathrm{w} / \mathrm{v})$ agarose gel (Promega) in $1 \times$ TAB buffer (50 mM Tris acetate, $1 \mathrm{mM}$ EDTA, $\mathrm{pH} 8 \cdot 0$ ) and visualized with ethidium bromide under UV light.

Cloning and sequencing of PCR products. The desired PCR products, which approximately matched nucleotide positions 250-840 in the HSP60 gene, were purified using the UNIQ-5 column DNA gel extraction kit (Sangon and NSBC) as recommended by the manufacturer. The purified fragments were ligated to pUCm-T (Sangon) with $\mathrm{T}_{4}$ DNA ligase $(\mathrm{MBI})$. The ligation products were then transformed into competent Escherichia coli DH5 $\alpha$, which was prepared by the $\mathrm{CaCl}_{2}$ method. Ampicillin $\left(100 \mu \mathrm{g} \mathrm{ml}^{-1}\right)$ and a bluewhite selection (Sambrook et al., 1989) were used to choose the required transformants, and the plasmids were extracted using the alkaline lysis method. To verify the presence of a correct insert of the partial HSP60 gene, PCR protocols, as 
Table 1. List of strains and GenBank accession numbers of partial HSP60 gene sequences of bifidobacteria and related bacterial strains

\begin{tabular}{|c|c|c|}
\hline Species & Strain & GenBank no. \\
\hline Bifidobacterium adolescentis & $\mathrm{JCM} 1275^{\mathrm{T}}$ & AF210319 \\
\hline Bifidobacterium angulatum & $\mathrm{JCM} 7096^{\mathrm{T}}$ & AF240568 \\
\hline Bifidobacterium animalis & JCM $1190^{\mathrm{T}}$ & AY004273 \\
\hline Bifidobacterium asteroides & $\mathrm{JCM} 8230^{\mathrm{T}}$ & AF240570 \\
\hline Bifidobacterium bifidum & $\mathrm{JCM} 1255^{\mathrm{T}}$ & AY004280 \\
\hline Bifidobacterium boum & $\mathrm{JCM} 1211^{\mathrm{T}}$ & AY004285 \\
\hline Bifidobacterium breve & JCM $1192^{\mathrm{T}}$ & AF240566 \\
\hline Bifidobacterium catenulatum & JCM $1194^{\mathrm{T}}$ & AY004272 \\
\hline Bifidobacterium choerinum & $\mathrm{JCM} 1212^{\mathrm{T}}$ & AY013247 \\
\hline Bifidobacterium coryneforme & $\mathrm{JCM} 5819^{\mathrm{T}}$ & AY004275 \\
\hline Bifidobacterium cuniculi & JCM $1213^{\mathrm{T}}$ & AY004283 \\
\hline Bifidobacterium denticolens & DSM $10105^{\mathrm{T}}$ & AF240565 \\
\hline Bifidobacterium dentium & JCM $1195^{\mathrm{T}}$ & AF240572 \\
\hline Bifidobacterium gallicum & $\mathrm{JCM} 8224^{\mathrm{T}}$ & AF240575 \\
\hline Bifidobacterium gallinarum & JCM $6291^{\mathrm{T}}$ & AY004279 \\
\hline Bifidobacterium indicum & $\mathrm{JCM} 1302^{\mathrm{T}}$ & AF240574 \\
\hline Bifidobacterium infantis & $\mathrm{JCM} 1222^{\mathrm{T}} \dagger$ & AF240577 \\
\hline Bifidobacterium infantis & JCM 1210 & AF 240576 \\
\hline Bifidobacterium infantis & Isolate BB52 & AY004288 \\
\hline Bifidobacterium infantis & Isolate 9901 & AY004286 \\
\hline Bifidobacterium inopinatum & DSM $10107^{\mathrm{T}}$ & AY004281 \\
\hline Bifidobacterium lactis & DSM $10140^{\mathrm{T}} \dagger$ & AY004282 \\
\hline Bifidobacterium lactis & Isolate 9950 & AY004287 \\
\hline Bifidobacterium lactis & Isolate 9952 & AF286735 \\
\hline Bifidobacterium longum & $\mathrm{JCM} 1217^{\mathrm{T}}$ & AF240578 \\
\hline Bifidobacterium magnum & $\mathrm{JCM} 1218^{\mathrm{T}}$ & AF240569 \\
\hline Bifidobacterium merycicum & $\mathrm{JCM} 8219^{\mathrm{T}}$ & AY004277 \\
\hline Bifidobacterium minimum & $\mathrm{JCM} 5821^{\mathrm{T}}$ & AY004284 \\
\hline Bifidobacterium pseudocatenulatum & JCM $1200^{\mathrm{T}}$ & AY004274 \\
\hline Bifidobacterium pseudolongum subsp. globosum & $\mathrm{JCM} 5820^{\mathrm{T}}$ & AF286736 \\
\hline Bifidobacterium pseudolongum subsp. pseudolongum & $\mathrm{JCM} 1205^{\mathrm{T}}$ & AF240573 \\
\hline Bifidobacterium pullorum & $\mathrm{JCM} 1214^{\mathrm{T}}$ & AY004278 \\
\hline Bifidobacterium ruminantium & $\mathrm{JCM} 8222^{\mathrm{T}}$ & AF240571 \\
\hline Bifidobacterium suis & JCM $1269^{\mathrm{T}}$ & AY013248 \\
\hline Bifidobacterium thermacidophilum & AS $1.2282^{\mathrm{T}}$ & AY004276 \\
\hline Bifidobacterium thermophilum & $\mathrm{JCM} 1207^{\mathrm{T}}$ & AF240567 \\
\hline Gardnerella vaginalis & ATCC $14018^{\mathrm{T}}$ & AF240579 \\
\hline Escherichia coli & $\mathrm{K}-12$ & AE $000487^{*}$ \\
\hline
\end{tabular}

* Previously reported GenBank sequence.

$\uparrow$ Strains in the same species used for the HSP60 sequence similarity analysis.

described above, were performed using approximately $50 \mathrm{ng}$ plasmid DNA as the template. Nucleotide sequencing was performed by TaKaRa using the ABI PRISM Big Dye Terminator Cycle Sequencing Ready Reaction kit (Perkin Elmer) and an ABI PRISM 377XL DNA sequencer. The T7 promoter (5'-TAA TAC GAC TCA CTA TAG GG-3') was used as the sequencing primer.

Analysis of sequence data, and phylogenetic tree construction. The partial HSP60 sequences determined in this study, and that of $E$. coli from the database, were aligned by the CLUSTAL W software (version 1.5). Continuous fragments of $538 \mathrm{bp}$, omitting the primer sequences, were used for analysis. The similarities were calculated and converted into a distance matrix with the Jukes-Cantor coefficient by the DNADIST program (PHYLIP, version 3.5; Felsenstein, 1993). A phylogenetic tree rooted with E. coli was constructed by the Fitch method (PHYLIP, version 3.5) and the topology of the tree was evaluated by 1000 trials of bootstrap analysis with the SEQUENCEBOOTS program (PHYLIP, version 3.5). Partial HSP60 DNA sequences were also translated into protein sequences by using the PRIMER PREMIER program, version 5.00 (Biosoft International). The protein sequence similarities were calculated and converted into a distance matrix with the DNAMAN program, version 4.0 (Lynnon Biosoft). 


\section{RESULTS}

\section{Cloned 600 bp partial HSP60 gene sequences of bifidobacteria}

A library of 37 cloned 600 bp HSP60 PCR products, representing 30 validly described Bifidobacterium species and subspecies and $G$. vaginalis, was generated. These PCR products were cloned and sequenced, and their GenBank accession numbers are listed in Table 1.

\section{Sequence similarity in partial HSP60 genes and proteins among different Bifidobacterium species and subspecies}

HSP60 DNA and protein sequence similarities among the four Bifidobacterium infantis and three Bifidobacterium lactis strains listed in Table 1 were determined. For the strains within the same species of $B$. infantis or B. lactis, the HSP60 DNA sequences were highly conserved, the similarity ranging from $99 \cdot 4$ to $100 \%$ (mean $99.5 \%$ ); the HSP60 protein sequence similarities were consistently $100 \%$.

At the interspecies level, HSP60 DNA sequence similarity among 30 different Bifidobacterium species ranged generally from 73 to $96 \%$ (mean $85 \%$ ), but was $98 \%$ for the two groups of closely related species, Bifidobacterium animalis and B. lactis, as well as $B$. infantis, Bifidobacterium longum and Bifidobacterium suis, whose 16SrRNA sequence similarities are all above $99 \%$. The HSP60 DNA sequence similarity between the two subspecies of $B$. pseudolongum, $B$. pseudolongum subsp. pseudolongum and B. pseudolongum subsp. globosum, was $96 \%$. The lowest similarity levels $(73 \%)$ were determined between $B$. inopinatum and Bifidobacterium cuniculi, B. inopinatum and $B$. pseudolongum subsp. pseudolongum, and $B$. inopinatum and $B$. pseudolongum subsp. globosum. It is noteworthy that both of $B$. denticolens and $B$.

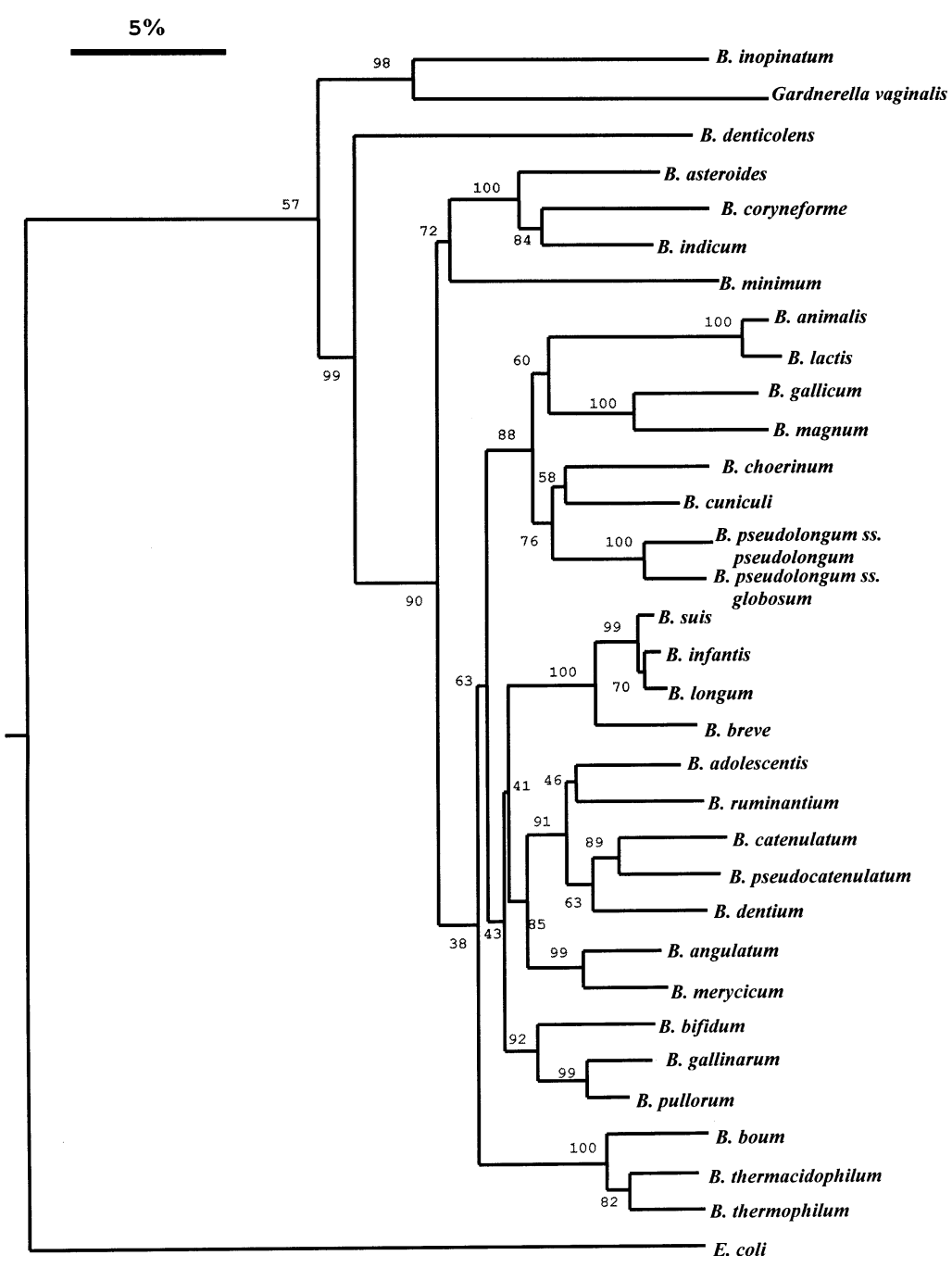

Fig. 1. Phylogenetic tree based on partial HSP60 DNA sequences. The tree was rooted with Escherichia coli and constructed using the Fitch method with bootstrap values calculated from 1000 trees. The number at each branch point represents the percentage bootstrap support. Bar, $5 \%$ sequence divergence. 
inopinatum showed exceptionally low similarities with respect to all of the other members of the genus Bifidobacterium (76-80\%, mean $78 \%$, and $73-78 \%$, mean $75 \%$, respectively). The HSP60 DNA sequence similarities between $G$. vaginalis and different Bifidobacterium species ranged from 71 to $79 \%$ (mean $74 \%$ ).

HSP60 protein sequence similarities among the 30 Bifidobacterium species were also examined. Because of the degeneracy of genetic codes, it is not surprising that the amino acid sequence similarity between any given pair was always higher than that of the corresponding DNA sequence. The amino acid sequence similarity at the interspecies level ranged from 83 to $100 \%$ (mean $92 \%$ ). The highest similarity was between B. animalis and B. lactis $(100 \%), B$. longum and B. suis $(100 \%)$, and among Bifidobacterium boum, Bifidobacterium thermacidophilum and Bifidobacterium thermophilum (all $100 \%$ ), and the lowest similarity was between Bifidobacterium merycicum and B. inopinatum $(83 \%)$. As expected, B. denticolens and $B$. inopinatum showed quite low amino acid sequence similarities with other Bifidobacterium species (84-92\%, mean $86 \% ; 83-92 \%$, mean $87 \%$, respectively). The amino acid sequence similarity between $G$. vaginalis and all the Bifidobacterium species studied ranged from 86 to $93 \%$ (mean $91 \%$ ).

\section{Phylogenetic analysis}

A phylogenetic tree rooted with E. coli was constructed using the Fitch method with bootstrap values calculated from 1000 trees (Fig. 1). On the basis of the HSP60 DNA sequences, the Bifidobacterium species were grouped into three clusters: most of the species (28 out of 30 ) were grouped into cluster $1, B$. denticolens formed cluster 2 , and B. inopinatum and $G$. vaginalis formed cluster 3 . The species in cluster 1 were further grouped into subclusters: Bifidobacterium indicum, Bifidobacterium coryneforme, Bifidobacterium asteroides (the three species isolated from the intestines of bees) and Bifidobacterium minimum formed one subcluster, while the three thermophilic species, $B$. thermophilum, B. thermacidophilum and B. boum, formed another one; the remaining 21 species were grouped into the third subcluster, having a profile quite similar to that determined on the basis of $16 \mathrm{~S}$ rRNA (Miyake et al., 1998).

\section{DISCUSSION}

\section{Inter- and intra-species sequence similarities of partial HSP60 versus the 16S rRNA gene of Bifidobacterium}

Because of the limitations of 16S rRNA analysis in the phylogenetic study of closely related bacterial taxa and the inconvenience of DNA-DNA hybridization, partial HSP60 DNA sequence analysis was conducted for most of the Bifidobacterium species described in this study. The result has shown that the partial HSP60 DNA sequence similarity among different Bifido- bacterium species ranged from 73 to $96 \%$ (mean $85 \%$ ). Exceptionally high similarities $(98 \%)$ were determined among the group comprising $B$. infantis, $B$. longum and $B$. suis, as well as between $B$. animalis and $B$. lactis. However, the 16S rRNA similarities of the two groups all reached levels above $99 \%$. By comparison, the $16 \mathrm{~S}$ rRNA sequence similarities among all the Bifidobacterium species ranged from 90.2 to $99.9 \%$ (mean $95 \%$ ) and were exceptionally high for five groups: (1) $B$. indicum and B. coryneforme (similarity 99.1\%), (2) Bifidobacterium catenulatum and Bifidobacterium pseudocatenulatum (similarity 99.5\%), (3) Bifidobacterium saeculare, Bifidobacterium gallinarum and Bifidobacterium pullorum group (similarity 99-3-99. $9 \%$ ), (4) B. longum, B. infantis and B. suis group (similarity $99 \cdot 1-99 \cdot 2 \%)$, (5) $B$. animalis and $B$. lactis (similarity 99\%) (Miyake et al., 1998). Although the HSP60 DNA sequence similarities of these groups were higher than the mean, the discriminatory power was still stronger than that of $16 \mathrm{~S}$ rRNA. For example, the HSP60 DNA sequence similarity was $91 \%$ between $B$. indicum and $B$. coryneforme, $93 \%$ between $B$. catenulatum and B. pseudocatenulatum, $96 \%$ between B. gallinarum and B. pullorum, $98 \%$ among B. longum, $B$. infantis and $B$. suis, and $98 \%$ between $B$. animalis and $B$. lactis. For the strains within the same species of $B$. infantis or B. lactis, the HSP60 DNA sequence similarities were $99 \cdot 4-100 \%$. Thus, analysis of partial HSP60 gene similarity is a powerful tool for the phylogenetic study of Bifidobacterium species, has a resolution power higher than that of $16 \mathrm{~S}$ rRNA homology analysis, and is much more convenient than DNA-DNA hybridization.

\section{Phylogenetic analysis of bifidobacteria and gardnerella by using partial HSP60 gene similarities}

In the phylogenetic tree based on 16S rRNA (Miyake et al., 1998), bifidobacteria were grouped into two clusters. The two species isolated from human dental caries, B. denticolens and B. inopinatum, formed cluster 1 ; all of the other members, including $G$. vaginalis, formed cluster 2 . In the present study, based on partial HSP60 gene similarities, a phylogenetic tree including the Bifidobacterium species and $G$. vaginalis was constructed (Fig. 1). The topology was basically similar to that of $16 \mathrm{~S}$ rRNA but with the following differences: (1) instead of clustering with most of the Bifidobacterium species in the $16 \mathrm{~S}$ rRNA phylogenetic tree, $G$. vaginalis formed a relatively independent branch with $B$. inopinatum, a similar low- $\mathrm{G}+\mathrm{C}$ Bifidobacterium species, which confirmed the genus status of genus Gardnerella; (2) B. denticolens, which formed one cluster with $B$. inopinatum in the $16 \mathrm{~S}$ rRNA tree, was in an independent branch of the HSP60 tree, indicating that the phylogenetic relationship between $B$. denticolens and $B$. inopinatum may be more distant than was estimated from 16S rRNA sequence similarity. Furthermore, the clustering of strains in the tree of the HSP60 gene was better correlated with the DNA base composition than that of $16 \mathrm{~S}$ rRNA. In the tree, 
all of the high- $\mathrm{G}+\mathrm{C}(55-67 \mathrm{~mol} \%)$ bifidobacteria were grouped into one compact cluster, whereas the only two low- $\mathrm{G}+\mathrm{C}$ members, $B$. inopinatum (45 $\mathrm{mol} \%$ ) and $G$. vaginalis (42 $\mathrm{mol} \%$ ), formed another cluster; $B$. denticolens $(55 \mathrm{~mol} \%)$ formed an intermediate cluster between the other two.

\section{HSP60 protein sequence similarities of bifidobacteria}

It is not surprising that the HSP60 protein sequence similarities should be higher than those of the corresponding DNA sequences. However, an interesting observation is shown for the group comprising $B$. thermophilum, B. thermacidophilum and B. boum. Although their HSP60 DNA did not show very high similarities (94-95\%), the HSP60 protein sequence similarities were all $100 \%$, the highest among all the Bifidobacterium species. It is suggested that there is some association between their thermophilic characteristics and the shared primary structures of their heat-shock proteins.

\section{Removal of $B$. denticolens and $B$. inopinatum from the genus Bifidobacterium}

It is noteworthy that, on the basis of HSP60 DNA sequences, $B$. denticolens and $B$. inopinatum were quite distant from other Bifidobacterium species. This result was in accordance with that of $16 \mathrm{~S}$ rRNA sequence homology analysis, and led to the view that these two organisms should be removed from the genus Bifidobacterium and proposed as new genera in the family Bifidobacteriaceae (Stackebrandt et al., 1997). Further studies, such as the analysis of other conserved molecules, are needed to confirm this view.

\section{ACKNOWLEDGEMENTS}

This study was supported by a grant from the China National Foundation of Sciences (no. 3007001) and a special grant for taxonomic studies from the Chinese Academy of Sciences.

\section{REFERENCES}

Crociani, F., Biavati, B., Alessandrini, A., Chiarini, C. \& Scardovi, V. (1996). Bifidobacterium inopinatum sp. nov. and Bifidobacterium denticolens sp. nov., two new species isolated from human dental caries. Int J Syst Bacteriol 46, 564-571.

Dong, X., Xin, Y., Jian, W., Liu, X. \& Ling, D. (2000). Bifidobacterium thermacidophilum sp. nov., isolated from an anaerobic digester. Int J Syst Evol Microbiol 50, 119-125.
Felsenstein, J. (1993). PHYLIP (Phylogeny Inference Package), version 3.5. Department of Genetics: University of Washington, Seattle, USA.

Gupta, R. S. (1995). Evolution of the chaperonin families (Hsp60, Hsp10 and Tcp-1) of proteins and the origin of eukaryotic cells. Mol Microbiol 15, 1-11.

Kamla, V., Henrich, B. \& Hadding, U. (1996). Phylogeny based on elongation factor Tu reflects the phenotypic features of mycoplasmas better than that based on 16SrRNA. Gene 171, 83-87. Kwok, A. Y. C., Su, S.-C., Reynolds, R. P., Bay, S. J., Av-Gay, Y., Dovichi, N. J. \& Chow, A. W. (1999). Species identification and phylogenetic relationships based on partial HSP60 gene sequences within the genus Staphylococcus. Int J Syst Bacteriol 49, 1181-1192.

Lauer, E. \& Kandler, O. (1983). DNA-DNA homology, murein types and enzyme patterns in the type strains of the genus Bifidobacterium. Syst Appl Microbiol 4, 42-64.

Marmur, J. (1961). A procedure for the isolation of deoxyribonucleic acid from microorganisms. J Mol Biol 3, 208-218.

Meile, L., Ludwig, W., Rueger, U., Gut, C., Kaufamnn, P., Dasen, G., Wenger, S. \& Teuber, M. (1997). Bifidobacterium lactis sp. nov., a moderately oxygen tolerant species isolated from fermented milk. Syst Appl Microbiol 20, 57-64.

Miyake, T., Watanabe, K., Watanabe, T. \& Oyaizu, H. (1998). Phylogenetic analysis of the genus Bifidobacterium and related genera based on 16S rDNA sequences. Microbiol Immunol 42, 661-667.

Rusanganwa, E., Singh, B. \& Gupta, R. S. (1992). Cloning of HSP60 (GroEL) operon from Clostridium perfringens using a polymerase chain reaction based approach. Biochim Biophys Acta 1130, 90-94.

Sambrook, J., Fritsch, E. F. \& Maniatis, T. (1989). Molecular Cloning: a Laboratory Manual, 2nd edn. Cold Spring Harbor: NY : Cold Spring Harbor Laboratory.

Scardovi, V. (1986). Genus Bifidobacterium Orla-Jensen. In Bergey's Manual of Systematic Bacteriology, vol. 2, pp. 1418-1434. Edited by P. H. A. Sneath, N. S. Mair, M. E. Sharpe \& J. G. Holt. Baltimore: Williams \& Wilkins.

Segal, G. \& Ron, E. Z. (1996). Regulation and organization of the groE and dnaK operons in Eubacteria. FEMS Microbiol Lett 138, $1-10$.

Stackebrandt, E. \& Goebel, B. M. (1994). A place for DNA-DNA reassociation and $16 \mathrm{~S}$ rRNA sequence analysis in the present species definition in bacteriology. Int $J$ Syst Bacteriol 44, 846-849.

Stackebrandt, E., Rainey, F. A. \& Ward-Rainey, N. L. (1997). Proposal for a new hierarchic classification system, Actinobacteria classis nov. Int J Syst Bacteriol 47, 479-491.

Viale, A. M., Arakaki, A. K., Soncini, F. C. \& Ferreyra, R. G. (1994). Evolutionary relationships among eubacterial groups as inferred from GroEL (chaperonin) sequence comparisons. Int $J$ Syst Bacteriol 44, 527-533. 